\title{
Template-Monomer Interaction in Molecular Imprinting: Is the Strongest the Best?
}

\author{
Xuewen Fu1,2, Qiang Yang2, Qiang Zhou², Qiang Lin ${ }^{*}$, Chun Wang2* \\ ${ }^{1}$ Chemistry \& Chemical Engineering College, Hainan Normal University, Haikou, China \\ ${ }^{2}$ Chengdu Institute of Biology, Chinese Academy of Sciences, Chengdu, China \\ Email: linqiang@hainnu.edu.cn, wangchun@cib.ac.cn
}

Received 12 March 2015; accepted 13 April 2015; published 14 April 2015

Copyright (C) 2015 by authors and Scientific Research Publishing Inc.

This work is licensed under the Creative Commons Attribution International License (CC BY). http://creativecommons.org/licenses/by/4.0/

(c) (i) Open Access

\begin{abstract}
Molecularly imprinted polymers (MIPs) represent a new class of materials possessing high selectivity and affinity for the target molecule. They have been utilized as sensors, catalysts, sorbents for solid-phase extraction, stationary phase for liquid chromatography, mimics of enzymes, receptors, and antibodies. In this research, molecular imprinted polymers (MIPs) for luteolin were prepared using acrylamide, 4-vinylpyridine and 1-allyl-piperazine as functional monomers and ethylene glycol dimethacrylate as cross-linker by non-covalent imprinting method. UV-visible spectra were used to evaluate the interaction strength between the template and the monomers. The composites of the polymers were calculated from elementary analysis. The porous properties of the imprinted polymers have been determined from nitrogen adsorption-desorption isotherms. The imprinting efficiency of the prepared MIPs was evaluated by selective adsorption for luteolin and its structural analogues. Although the interaction strength of monomers to the template was in the order 1-ALPP > 4-VP > AA, the binding affinity of the imprinted polymers towards luteolin was in the order MIP $2>$ MIP $3>$ MIP 1. Our results demonstrated that the imprinting efficiency was depending not only on the interaction strength between the template and the monomer, but also on the fidelity in transferring the complex into the polymer.
\end{abstract}

\section{Keywords}

Molecular Imprinting, Luteolin, Polymerization, Efficiency

\section{Introduction}

Molecular imprinting has been widely used for the development of tailor-made receptor binding sites in a three-

${ }^{*}$ Corresponding author.

How to cite this paper: Fu, X.W., Yang, Q., Zhou, Q., Lin, Q. and Wang, C. (2015) Template-Monomer Interaction in Molecular Imprinting: Is the Strongest the Best? Open Journal of Organic Polymer Materials, 5, 58-68.

http://dx.doi.org/10.4236/ojopm.2015.52007 
dimensional, cross-linked polymer matrix. Molecularly imprinted polymers (MIPs) are thus generated with ligand-specific recognition properties analogous to biological systems, and have gained considerable attention in applications, such as solid-phase extraction [1] [2], chiral compounds isolation [3]-[5], sensors [6], catalysis [7][10] and synthetic auxiliary [11] [12].

In the case of molecularly imprinted polymers prepared using non-covalent interactions, a key step in the polymer synthesis is the formation of the complex between the template and functional monomer(s). It is commonly accepted that the stronger the interactions between the template and monomers are, the more stable of the host-guest complex prior to polymerization is [13], and consequently the better imprinting efficiency of the resulted polymers is. Many theoretical [14] and experimental studies [15]-[17] focused on the formation and stability of pre-polymerization complexes. However, the fidelity of MIPs could be affected by many factors, such as the relative reactivity of the functional monomers and cross-linkers, the solvents, reaction conditions, etc., in the polymerization step. Binding experiments can be used to evaluate the polymer performance, providing information about the polymer capacity, selectivity, affinity, etc. Texture properties of imprinted polymers may reflect the physical and chemical events in the polymerization process. There are little reports emphasized on the later. In order to understand the influence of physical and chemical events during polymerization process on the performance of MIPs, both texture structure study and binding experiments evaluations are necessary.

Luteolin, 3',4',5,7-tetrahydroxyflavone, is a common flavonoid that exists along with other structural analogue flavonoids, such as rutin, genestine, etc., in many types of plants including fruits [18], vegetables [19], and medicinal herbs [20]. Plants rich in luteolin have been used in Chinese traditional medicine for treating various diseases such as hypertension, inflammatory disorders, and cancer. Having multiple biological effects such as anti-inflammation, anti-allergy and anticancer, luteolin functions as either an antioxidant or a pro-oxidant biochemically. However, due to the occurrence of luteolin in natural resources in low concentrations, and because of the structural similarity of the co-existing flavonoids, new selective materials employing molecular recognition mechanisms may enable selective isolation from complex samples.

This paper addresses the importance of understanding the factors influencing the imprinting efficiency in the polymerization step during MIP preparation. Luteolin, a polyphenol flavonoid, is chosen as the template. The polymers were prepared by using three functional monomers, namely, acrylamide (AA), 4-vinyl pyridine (4-VP) and 1-allypiperazine (1-ALPP), respectively. THF was as porogen, and ethylene glycol dimethycrylate (EGDMA) was as cross-linker. The texture properties were investigated by elementary analysis and $\mathrm{N}_{2}$ gas adsorption/desorption experiments. The correlation between the texture characteristics and the imprinting efficiency was also evaluated by binding tests.

\section{Experimental}

\subsection{Materials and Chemicals}

Luteolin, Genistein, Rutin, and Pyrene were purchased from Sigma-Aldrich. Acrylamide (AA) and 4-vinyl pyridine (4-VP) were supplied from Shanghai Aladdin Industrial Corporation. 1-allylpiperazine (1-ALPP) and ethylene glycol dimethacrylate (EGDMA) were obtained from Guangdong J\&K Scientific Ltd. Analytical-grade acetonitrile (ACN), tetrahydrofuran (THF) and glacial acetic acid, were purchased from Sichuan Xilong Chemical Co., Ltd.

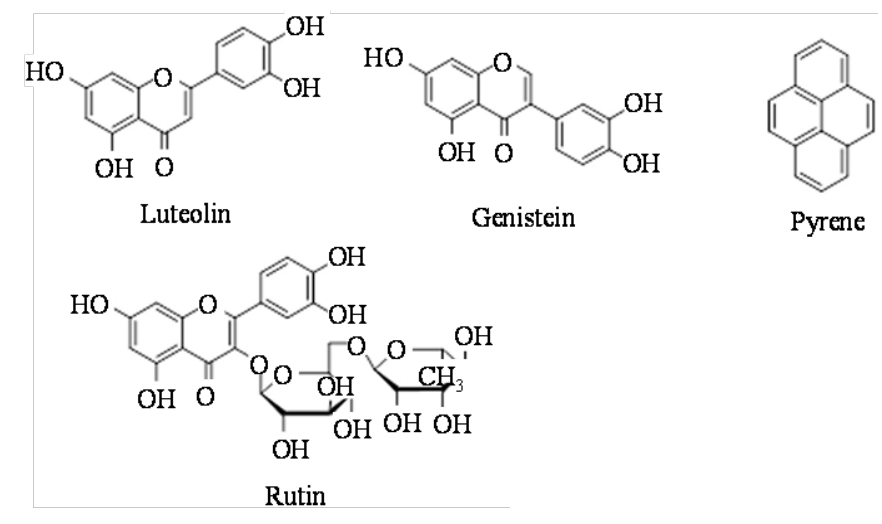




\subsection{Instrumentations}

The Fourier transform infrared (FTIR) spectra of the prepared polymers were obtained by using a Perkin Elmer Spectrum One FTIR spectrometer in the spectral range of $4000-400 \mathrm{~cm}^{-1}$ at a spectral resolution of $1 \mathrm{~cm}^{-1}$. UV-Vis spectra [21] were performed using a Perkin Elmer Ultraviolet-visible spectrophotometer. Euro EA3000 was used for elementary analysis. The nitrogen adsorption-desorption isotherms at $77 \mathrm{~K}$ were measured using a HYA2010-B2 instrument supplied by Beijing Zhongke Huiyu corporation of science and technology. Before the adsorption measurements, samples were degassed at $200^{\circ} \mathrm{C}$ for a period of $2 \mathrm{~h}$ prior to analysis. The data were evaluated with the Brunauer-Emmett-Teller (BET) and Barrett-Joyner-Halenda (BJH) methods to calculate the surface area and pore volume/pore size distribution, respectively.

\subsection{Preparation of the Molecularly Imprinted Polymers}

Imprinted (MIP) and non-imprinted (NIP) polymers were prepared via bulk polymerization in presence of a porogenic solvent. Briefly, $0.4 \mathrm{mmol}$ template (luteolin) and $2.4 \mathrm{mmol}$ functional monomers (AA, 4-VP and 1ALPP) were dissolved separately in $4.0 \mathrm{ml}$ of THF. Then 12.0 mmol EGDMA (cross-linker) and $20 \mathrm{mg}$ 2,2'Azobis(2-methylpropionitrile) (AIBN) were added to the prepared solutions. The pre-polymerization solution was sonicated for $5 \mathrm{~min}$ and then deoxygenated under nitrogen for $5 \mathrm{~min}$. The flask was sealed and the polymerization was carried out at $60^{\circ} \mathrm{C}$ for $24 \mathrm{~h}$ in a thermostat-control oil bath, until the polymerization was completed. Then, the bulk polymer obtained was grounded into powder with a mortar. The particles with diameters ranging from 25 to $45 \mu \mathrm{m}$ were collected through a 100 sieve. The template molecules were removed with a Soxhlet apparatus using methanol/glacial acetic acid mixture 90/10 (v/v). Then, particles were rinsed with methanol to eliminate residual acetic acid, dried under vacuum and stored at ambient temperature. The resulting imprinted polymers from monomers AA, 4-VP and 1-ALPP were assigned as MIP1, MIP2 and MIP3, respectively. Nonimprinted polymers were prepared via the same procedure but in the absence of template, and assigned as NIP1, NIP2 and NIP3 correspondingly.

\subsection{Spectroscopic Analysis of Interaction between Luteolin and Monomers}

The analysis was conducted by adding functional monomer solutions (AM, 4-VP and 1-ALPP) in the range of $2.8 \times 10^{-2}$ to $22.4 \times 10^{-2} \mathrm{mM}$ into a constant concentration of luteolin solution $\left(2.8 \times 10^{-2} \mathrm{mM}\right)$ in acetonitrile. After equilibrating for $30 \mathrm{~min}$, the changes of absorbance of these solutions were recorded at $338 \mathrm{~nm}$ with corresponding functional monomer solutions as the reference.

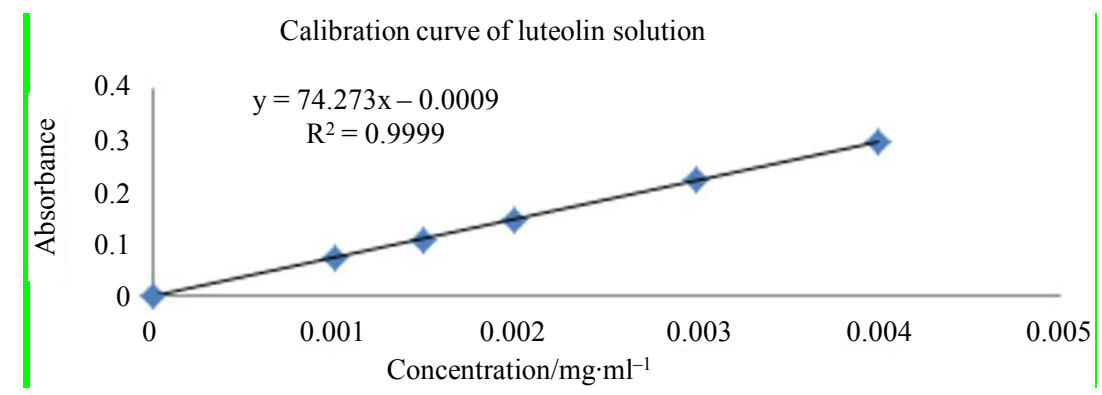

The stoichiometry of template-functional monomer complex was determined by Job's plot methods [22]. A series of acetonitrile solutions containing luteolin and 1-ALPP were prepared such that the sum of the total concentration remained constant $(0.1 \mathrm{mM})$. The mole fraction $(\mathrm{X})$ of 1-ALPP was varied from 0.1 to 1.0. The absorbance of luteolin without addition of 1-ALPP was denoted as $\mathrm{A}_{0}$, and the absorbance of the solution containing luteolin and 1-ALPP were denoted as A. The corrected absorbance $\left(\mathrm{A}_{0}-\mathrm{A}\right)$ at $338 \mathrm{~nm}$ was plotted against the molar fraction of the 1-ALPP solution.

\subsection{Adsorption and Selectivity Evaluation}

The equilibrium adsorption experiments were performed to evaluate the binding capability of MIPs and NIPs particles. The dried polymer particles $(20 \mathrm{mg})$ were placed in a $10 \mathrm{ml}$ centrifuge tube and mixed with $5.0 \mathrm{ml}$ of 
luteolin standard solutions at different concentrations $(0.01,0.02,0.04,0.06,0.1,0.2$ and $0.4 \mathrm{mM})$. Then, the tube was sealed and oscillated by a constant temperature oscillator at room temperature for $12 \mathrm{~h}$. After the binding process was completed, the mixture was filtrated by $0.45 \mu$ micro-filter. The concentration of free luteolin in the supernatant was determined by measuring the absorbance at $338 \mathrm{~nm}$. The amount of luteolin bound to the polymers $(\mathrm{Q})$ was calculated by subtracting the amount of free substrate from the initial luteolin concentration.

The binding selectivity evaluation was carried out with different structural analogues of luteolin: genistein, rutin and pyrene. The dried polymer particles $(20 \mathrm{mg})$ were placed in a $10 \mathrm{ml}$ centrifuge tube and mixed with 5.0 $\mathrm{ml}$ of different analogues standard solutions. Then the polymers were treated in the same way as described above. To make a comparison for imprinting effect, we define the specific adsorption values as IF = QMIP/ QNIP, where QMIP and QNIP are the amounts of bound template on the imprinted and non-imprinted polymers. The relative adsorption capacity for all materials to each of targets can be judged by bound-to-free $(\mathrm{B} / \mathrm{F})$ ratio value, which was calculated by the following equation: $\mathrm{B} / \mathrm{F}=\left(\mathrm{C}_{0}-\mathrm{C}\right) / \mathrm{C}$, where $\mathrm{C}_{0}(\mathrm{mmol} / \mathrm{L})$ and $\mathrm{C}(\mathrm{mmol} / \mathrm{L})$, are the concentration before and after adsorption, respectively.

\section{Results and Discussions}

\subsection{Preparation of MIPs}

The imprinted polymers were prepared by following the process demonstrated in Scheme 1:1) formation of prepolymerization complex of the template (luteolin) and functional monomers (AA, 4-VP or 1-ALPP) through non-covalent interactions; 2) polymerization of the functional monomers and cross-linker (EGDMA) with an initiator (AIBN); 3) removal of the template by Soxhlet extraction with methanol/glacial acetic acid mixture 90/10 $(\mathrm{v} / \mathrm{v})$.

\subsection{UV Analysis}

Presumably, the strength and structure of the assembled complex between functional monomer(s) and template determine the subsequent affinity and selectivity of the polymers. Thus, it is important to search for monomers that interact strongly with the template and form stable host-guest complexes prior to the polymerization. In this study, UV-vis spectroscopic method is used to evaluate the interaction of acrylamide (AA), 4-vinyl pyridine (4-VP) and 1-allypiperazine (1-ALPP) monomers at different molar concentrations with luteolin, as illustrated in Figure 1.

As clearly seen from Figure 1, by increasing the concentration of 1-ALPP, the maximum absorbance at 345 $\mathrm{nm}$ (due to $\pi-\pi$ transition of luteolin) decrease drastically. In case of 4-VPmonomer, less changes in maximum absorbance at 345 was observed. On the other hand, undetectable changes was noticed in case of AA monomer, indicating that the interaction strength between luteolin and the investigated monomers can be arranged in the following order: 1-ALPP $>4$-VP $>$ AA.

Job's plot analysis (Figure 2) of the complexation demonstrated a 1:1 complex between the functional monomer (1-ALPP) and the template (luteolin). The structure of the complex is assumed as in Figure 3.

\subsection{IR Analysis}

We take the IR spectra of three polymermaterials prepared with 1-ALPP as functional monomer before template removal (MIP3-as), after template removal (MIP3) and non-imprinted polymer (NIP3), as an example, to elucidate the imprinting process (Figure 4). The common broadpeak at $3449 \mathrm{~cm}^{-1}$ in all three spectra is attributed to the stretching vibration of the $\mathrm{N}-\mathrm{H}$ groups. The bands at $2950 \mathrm{~cm}^{-1}$ are assigned to the $\mathrm{C}-\mathrm{H}$ stretching vibrations. Comparison of MIP3-as with MIP3 and NIP3, the bands raised at 1630 is due to $\mathrm{C}=\mathrm{C}$ stretching vibrations of the luteolin aromatic ring. The band at $1461 \mathrm{~cm}^{-1}$ of the FTIR spectrum of MIP3-as is assigned to N-H in plane bending vibrations of the functional monomer. The increasing intensity around $1461 \mathrm{~cm}^{-1}$ and slightly shifted to higher wavenumber for MIP3-as is probably due to the formation of $-\mathrm{NH}^{3+}$ moiety between amino group and phenoxyl group of luteolin [23]. The peak at $1162 \mathrm{~cm}^{-1}$ is C-N stretching vibrations. In addition, the spectrum shows the presence of aromatic groups at 961 and $755 \mathrm{~cm}^{-1}$. The above results indicate that the template molecules are included in MIP3-as and functional monomers containing amino groups were successfully incorporated in all three polymers. In addition, the FTIR patterns of MIP3 are similar with those of non-imprinted material (NIP3), indicating the complete removal of luteolin from MIP3-as. 


\section{的占}

Functional monomer

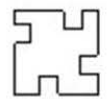

Template

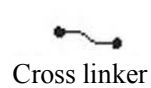

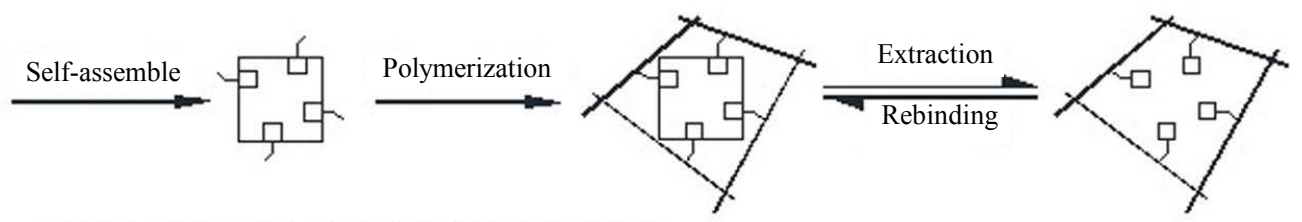

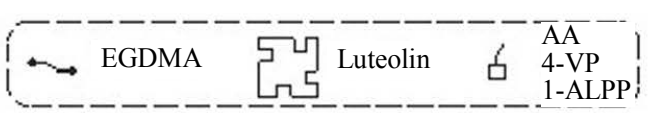

Scheme 1. Schematic representation for the process of MIPs preparation and rebinding.

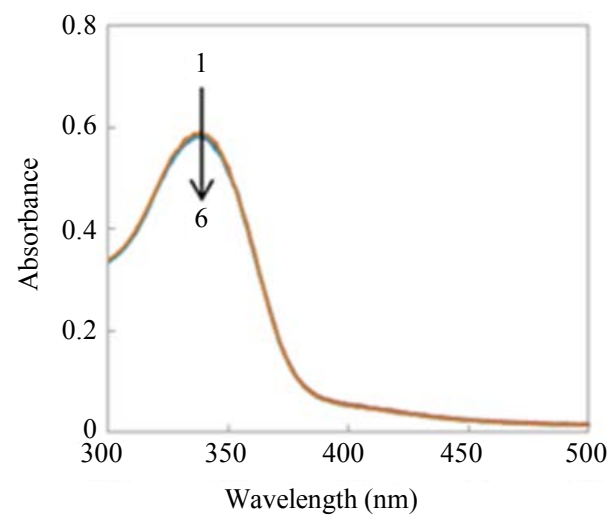

(a)

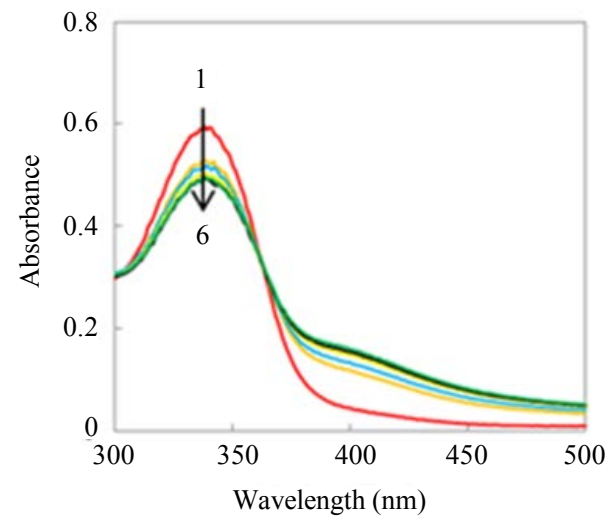

(b)

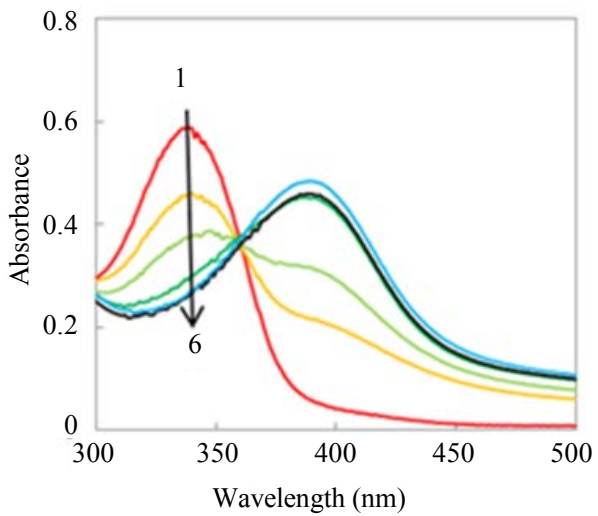

(c)

Figure 1. UV-Vis spectra of Luteolin in the presence of different concentrations of monomers in acetonitrile: (a) AM; (b) 4-VP; (c) 1-ALPP. Concentration of Luteolin: $2.8 \times 10^{-2} \mathrm{mM}$; Concentration of monomers for lines $1-6: 0,2.8 \times 10^{-2}, 5.6$ $\times 10^{-2}, 11.2 \times 10^{-2}, 16.8 \times 10^{-1}$, and $22.4 \times 10^{-1} \mathrm{mM}$, respectively.

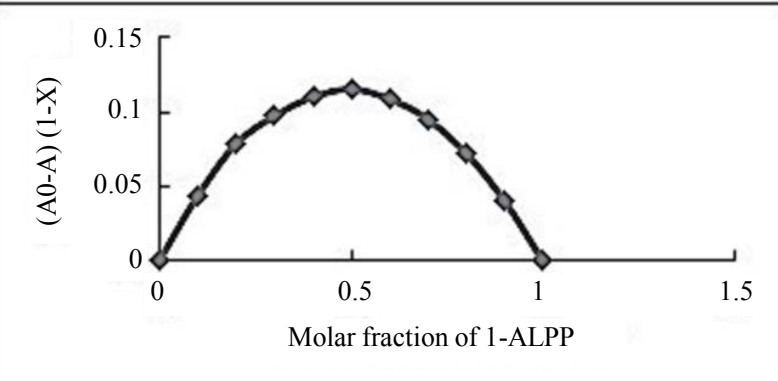

Figure 2. Job's plot of the complexation between 1-ALPP and luteolin. 


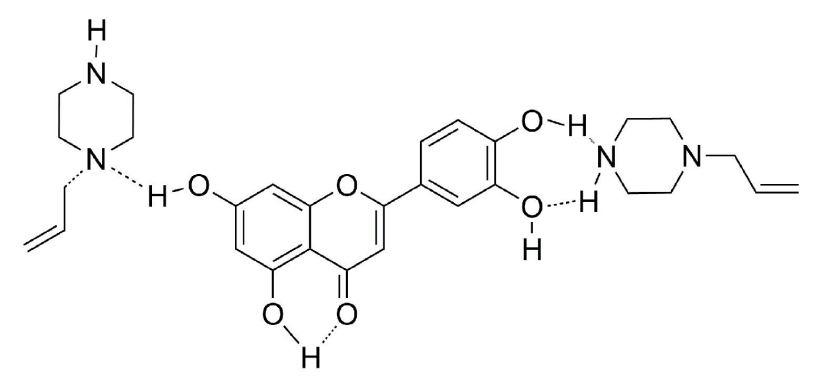

Figure 3. The proposed complex formation between Luteolin and 1-ALPP monomer.

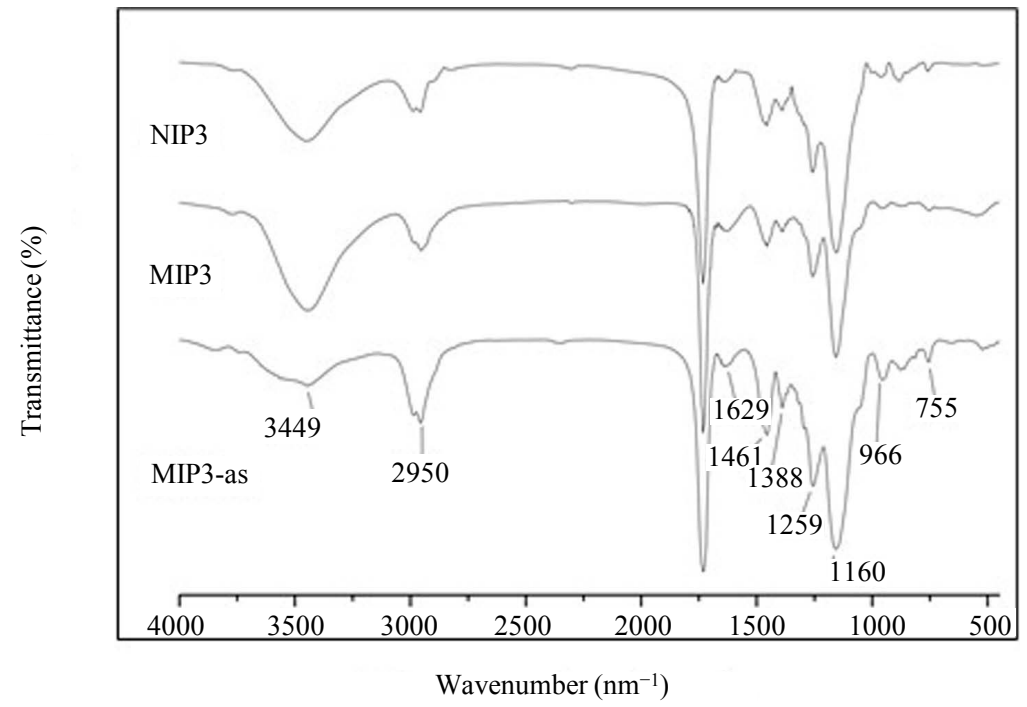

Figure 4. IR spectra of the luteolin MIPs and corresponding NIPs.

\subsection{Elementary Analysis}

As a complementary technique, elementary analysis for nitrogen contents was performed to identify the incorporation of functional monomers into the imprinted polymer. The results are summarized in Table 1.

Data in Table 1, column 3 and 4 represent the molar ratio of functional monomer/cross linker added and obtained by elementary analysis, respectively, which indicate that MIP 1 and NIP 1, and MIP 2 and NIP 2 contain almost the same amount of functional monomers AM and 4-VP, respectively. On the other hand, nitrogen content of NIP 3 is lower than that of MIP 3. The addition of template in MIP 3 increased the nitrogen content as compared to its corresponding non-imprinted materials. It is worth to mention that 1-ALPP contains two nitrogen atoms in each 1-ALPP molecule rather than one as in AM and 4-VP. Thus, MIP 3 contains only $50 \%$ of functional monomer corresponding to MIP 1 and MIP 2. Allyl monomers are known to retard polymerization [24]. They produce dead polymer chains due to $\mathrm{N}$ radicals via hydrogen abstraction which are less reactive than propagating radicals. The addition of luteolin leads to the protonation of the nitrogen on 1-ALPP, preventing the Habstraction, and consequently increasing their reactivity, but still less reactive than other propagating radicals.

\section{5. $\mathrm{N}_{2}$ Adsorption/Desorption Measurements}

The porous structures of MIPs are formed due to the template-functional monomer induced cavities and the texture voids formed by solvents among cross-links. The $\mathrm{N}_{2}$ adsorption/desorption isotherm and BJH pore size distribution graphs are shown in Figure 5.

The prepared polymers all exhibit "type IV" isotherm (Figure 5), according to IUPAC classifications, which are related to mesoporous materials. The fact that all imprinted and non-imprinted polymers gave a peak center around $4 \mathrm{~nm}$, suggested that this dimension of pores were probably generated during the polymerization due to 
Table 1. Elementary analysis of prepared materials.

\begin{tabular}{cccc}
\hline \multirow{2}{*}{ Sample Code } & Nitrogen Content (\%) & \multicolumn{2}{c}{ Functional Monomer/Cross Linker } \\
\cline { 3 - 4 } MIP 1 & 1.76 & Used & Integrated \\
NIP 1 & 1.76 & $1: 5$ & $0.97: 5$ \\
MIP 2 & 2.06 & $1: 5$ & $0.97: 5$ \\
NIP 2 & 1.84 & $1: 5$ & $1.03: 5$ \\
MIP 3 & 2.00 & $1: 5$ & $1.02: 5$ \\
NIP 3 & 1.38 & $1: 5$ & $0.65: 5$ \\
\hline
\end{tabular}

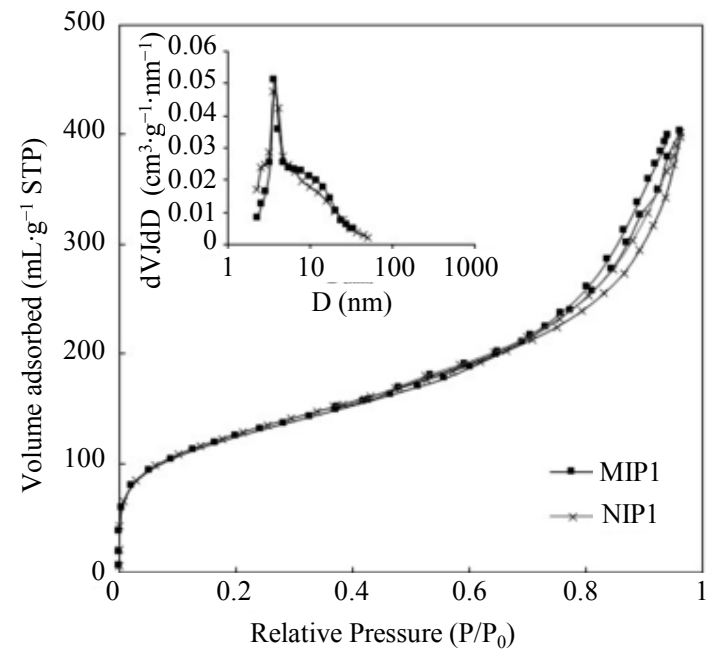

(a)

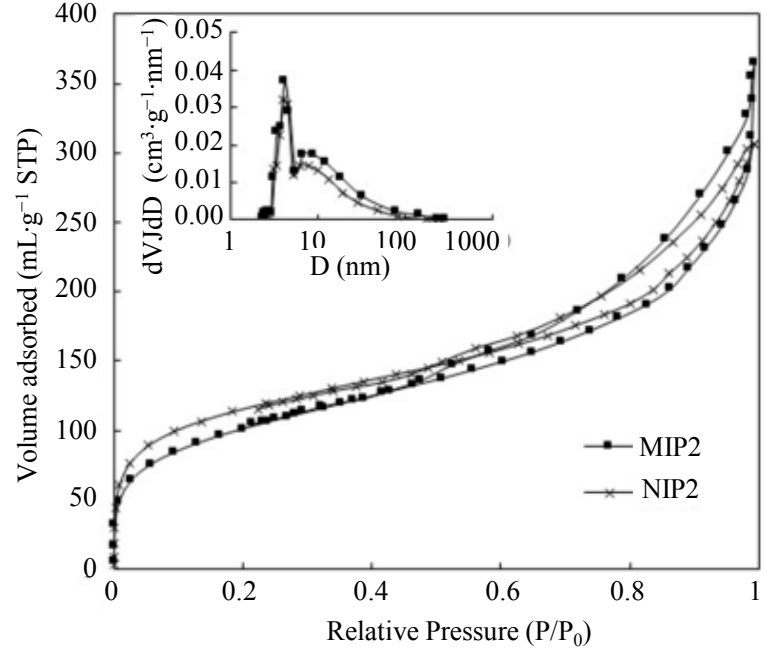

(b)

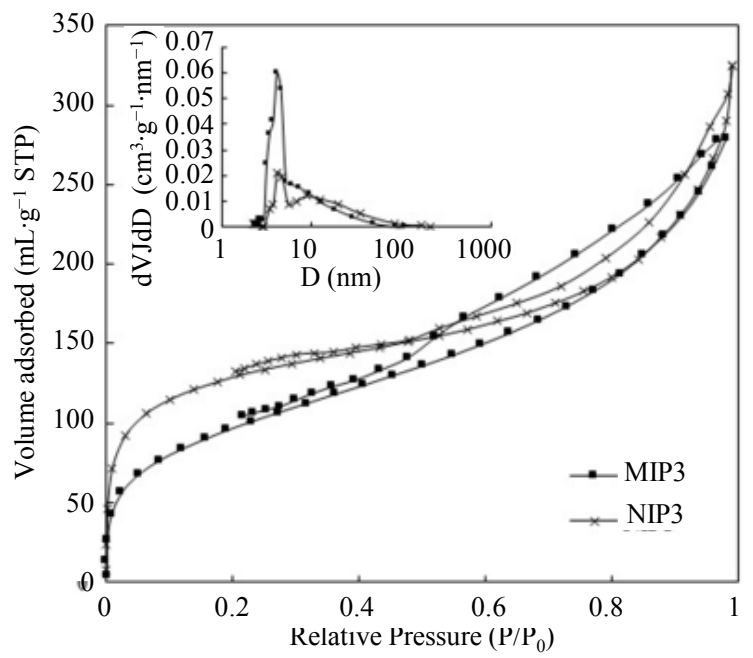

(c)

Figure 5. Nitrogen adsorption/desorption isotherm and pore size distribution.

the presence of porogens (solvents). For MIP1, very weak interactions between functional monomers and template do not appreciably form uniform new voids during polymerization process, as can be seen from the overlap of imprinted and non-imprinted on the pore size distribution (Figure 5(a)). On the other hand, for MIP3, very strong interactions may result in the ionic complex insoluble in the pre-polymerization solution, causing incom- 
plete polymerization between the monomers complexed on the template and cross-linkers. After washing, the unreacted monomers along with the templates were removed, left voids with heterogeneous functionality and pore sizes with broad distribution (Figure 5(c)). The pore size distribution curve shows no maxima; indicating a very wide distribution of pore size, resulted from the removal of different compositions of monomer-template complexes. Only MIP2, made with 4-VP as functional monomer, formed a distinguish peak around $10 \mathrm{~nm}$, revealed the formation of new pores by addition of the templates during polymerization (Figure 5(b)).

The porous properties of the imprinted and non-imprinted polymers were summarized in Table 2.

As clearly seen in Table 2, BET surface areas, BJH pore volumes and pore sizes are not changed for MIP 1 and NIP 1. BET surface area of both 4-VP and 1-ALPP prepared polymers (MIP 2 and MIP 3) were decreased after imprinting. But for MIP 2, both BJH pore volume and pore size were increased after imprinting, for MIP 3, $\mathrm{BJH}$ pore volume was decreased and the pore size increased. The reduced $\mathrm{BJH}$ pore volume might be due to the more condensed aggregates formed after incorporation of luteolin than non-imprinted polymer.

\subsection{Binding Capacity and Selectivity}

The monomers are the most important factors which influence the binding site affinity in the prepared molecularly imprinted polymer. Thus, it is widely accepted that the presence of specific monomer-template interactions is the basis for ligand (template) selective recognition sites in the resultant polymer. The template is assumed to interact by a combination of electrostatic interactions, $\pi-\pi$ interactions and hydrogen bonds with monomer prior to polymerization, and after polymerization with the functional groups of the polymer matrix [25]. However, the extent of template-monomer complexation is influenced after polymerization by the number of monomers attached and the availability of the functional groups on the monomers.

MIP 1 and NIP 1 have lower affinity for luteolin, which are consistent with the situation in solution. However, Figure 6 shows that MIP 2 adsorbs more luteolin than MIP 3, although MIP 3 had stronger interaction with luteolin in solution. This might be attributed to the following reasons: 1) in solution, one 1-ALPP interacts with two luteolin, (Figure 3), after polymerization, the allyl attached $\mathrm{N}$ may not be available for interacting with luteolin, due to steric hindrance; 2) the amount of monomer in MIP 3 is only half of that in MIP 2. Thus, less imprinted sites exist in MIP 3 than in MIP 2, as can be seen from elementary analysis, only $60 \%$ of monomers were incorporated in MIP 3 while almost $100 \%$ of added 4-VP were existed in the final MIP 2.

The imprinting effects were further evaluated by selective adsorption for the template and its structural analogues, genistein, rutin and pyrene, as illustrated in Figure 7. Although genistein and luteolin have the same molecular formulae, and the same amount of functional groups, but with different placement of phenyl substitute on chromene, the imprinted polymers had slightly higher adsorption for genistein than non-imprinted polymers. The selective adsorption of the imprinted polymers towards luteolin and genistein were: MIP $2>$ MIP $3>$ MIP 1 . We interpreted these results as the imprinting effects. Since more pre-polymerization complexes were successfully transferred into MIP 2 than in MIP 3, as explained previously by elementary analysis and nitrogen adsorption/desorption parameters. MIP 2 showed higher selectivity to the imprint molecule (luteolin) than its analogue (genistein). All the MIPs adsorbed more structural analogues of luteolin than the NIPs, probably due to the more cavities (bigger pore size, surface area and pore values) existing in the former. In addition, all the MIPs and NIPs showed very little selective adsorption for rutin and pyrene, because the size of these two molecules is far beyond the imprinting molecule. All the polymers had slightly higher affinity for rutin than pyrene, though the size of rutin is bigger than pyrene, because rutin bears hydroxyl groups which were interactive with the functional monomers inside the polymers.

Table 2. Physical properties of MIPs and NIPs characterized by nitrogen desorption porosimetry.

\begin{tabular}{cccc}
\hline Polymer & BET Surface Area $\left(\mathrm{m}^{2} \cdot \mathrm{g}^{-1}\right)$ & BJHpore Volume $\left(\mathrm{cm}^{3} \cdot \mathrm{g}^{-1}\right)$ & Pore Size $(\mathrm{nm})$ \\
\hline MIP1 & 451.70 & 0.401 & 3.73 \\
NIP1 & 457.07 & 0.404 & 3.50 \\
MIP2 & 370.48 & 0.564 & 6.50 \\
NIP2 & 418.37 & 0.474 & 5.12 \\
MIP3 & 357.08 & 0.431 & 5.32 \\
NIP3 & 454.23 & 0.502 & 4.35 \\
\hline
\end{tabular}




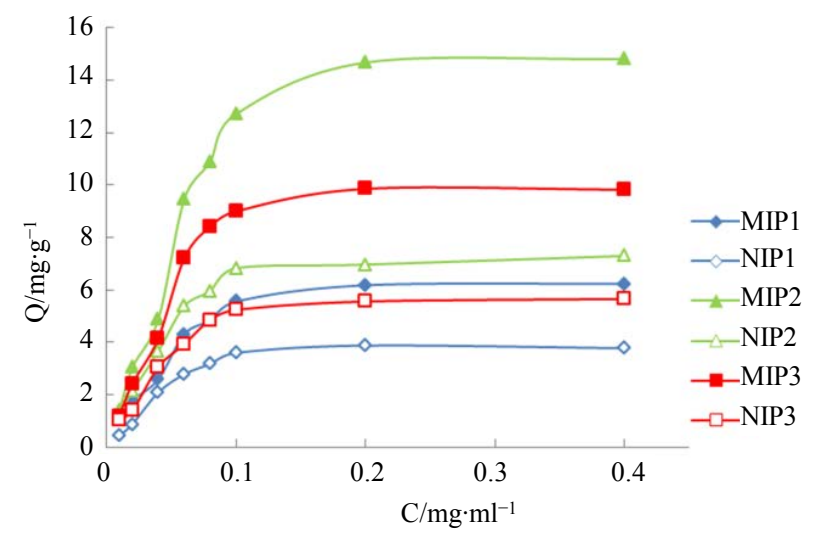

Figure 6. Binding isotherm of imprinted and non-imprinted polymers for luteolin.

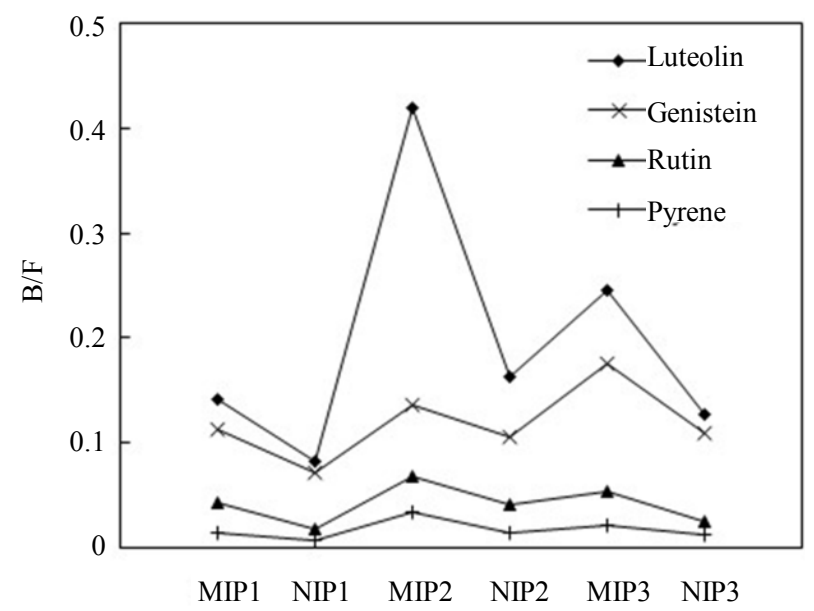

Figure 7. Selective adsorption of MIPs and NIPs for Luteolin, Genistein, Rutin and Pyrene.

\section{Conclusion}

We have demonstrated that the imprinting efficiency of molecular imprinting polymers depends not only on the stability of the pre-polymerization complex, but also on the fidelity in transferring the complex into the polymer. In other words, the polymerization process should not change the complexation between the template and monomers. Our findings suggest that the solubility of the pre-polymerization complex between the template and monomers must be taken into account in order to obtain effective imprinting. These results are fundamental in understanding the mechanism of imprinting and in designing and preparation of MIPs with high selectivity and performance.

\section{Acknowledgements}

This work was financially supported by the National Natural Science Foundation of China (NSFC21442012) and fund from Chemistry \& Chemical Engineering College, Hainan Normal University.

\section{References}

[1] Chen, S., Xing, X.H., Huang, J.J. and Xu, M.S. (2011) Enyzme-Assisted Extraction of Flavonoids from Ginkgo biloba Leaves: Improvement Effect of Flavonol Transglycosylation Catalyzed by Penicillium decumbens Cellulase. Enzyme and Microbial Technology, 48, 100-105. http://dx.doi.org/10.1016/j.enzmictec.2010.09.017

[2] Wang, J., Cormack, P.A.G., Sherrington, D.C. and Khoshdel, E. (2003) Monodisperse, Molecularly Imprinted Polymer 
Microspheres Prepared by Precipitation Polymerization for Affinity Separation Applications. Angewandte Chemie International Edition, 42, 5336-5338. http://onlinelibrary.wiley.com/doi/10.1002/anie.200352298/abstract

[3] Ansell, R.J. (2005) Molecularly Imprinted Polymers for the Enantioseparation of Chiral Drugs. Advanced Drug Delivery Reviews, 57, 1809-1835. http://dx.doi.org/10.1016/j.addr.2005.07.014

[4] Kempe, M. and Mosbach, K. (1995) Molecular Imprinting Used for Chiral Separations. Journal of Chromatography A, 694, 3-13. http://dx.doi.org/10.1016/0021-9673(94)01070-U

[5] Adbo, K., Andersson, H.S., Ankarloo, J., Karlsson, J.G., Norell, M.C., Olofsson, L., Svenson, J., Örtegren, U. and Nicholls, I.A. (1999) Enantioselective Tröger's Base Synthetic Receptors. Bioorganic Chemistry, 27, 363-371. http://dx.doi.org/10.1006/bioo.1999.1136

[6] Ersöz, A., Diltemiz, S.E., Özcan, A.A. and Denizli, A. (2008) Synergie between Molecular Imprinted Polymer Based on Solid-Phase Extraction and Quartz Crystal Microbalance Technique for 8-OHdG Sensing. Biosensors and Bioelectronics, 24, 742-747. http://dx.doi.org/10.1016/j.bios.2008.06.058

[7] Wulff, G., Sarhan, A. and Zabrocki, K. (1973) Enzyme-Analogue Built Polymers and Their Use for the Resolution of Racemates. Tetrahedron Letters, 14, 4329-4332. http://dx.doi.org/10.1016/S0040-4039(01)87213-0

[8] Lee, J., Bernard, S. and Liu, X.C. (2009) Nanostructured Biomimetic Catalysts for Asymmetric Hydrogenation of Enamides Using Molecular Imprinting Technology. Reactive and Functional Polymers, 69, 650-654. http://dx.doi.org/10.1016/j.reactfunctpolym.2009.04.006

[9] Huang, J.T., Zheng, S.H. and Zhang, J.Q. (2004) Molecularly Imprinting of Polymeric Nucleophilic Catalysts Containing 4-Alkylaminopyridine Functions. Polymer, 45, 4349-4354. http://dx.doi.org/10.1016/j.polymer.2004.03.050

[10] Visnjevski, G., Schomäcker, R., Yilmaz, E. and Brüggemann, O. (2005) Catalysis of a Diels-Alder Cycloaddition with Differently Fabricated Molecularly Imprinted Polymers. Catalysis Communications, 6, 601-606. http://dx.doi.org/10.1016/j.catcom.2005.06.001

[11] Wulff, G. and Vietmeier, J. (1989) Enzyme-Analogue Built Polymers, 25. Synthesis of Macroporous Copolymers from $\alpha$-Amino Acid Based Vinyl Compounds. Die Makromolekulare Chemie, 190, 1717-1726. http://onlinelibrary.wiley.com/doi/10.1002/macp.1989.021900723/abstract http://dx.doi.org/10.1002/macp.1989.021900723

[12] Bystroem, S.E., Boerje, A. and Akermark, B. (1993) Selective Reduction of Steroid 3- and 17-Ketones Using Lithium Aluminum Hydride Activated Template Polymers. Journal of the American Chemistry Society, 115, 2081-2083. http://pubs.acs.org/doi/abs/10.1021/ja00058a089 http://dx.doi.org/10.1021/ja00058a089

[13] Hashim, S.N.N.S., Boysen, R.I., Schwarz, L.J., Danylec, B. and Hearn, M.T.W. (2014) A Comparison of Covalent and Non-Covalent Imprinting Strategies for the Synthesis of Stigmasterol Imprinted Polymers. Journal of Chromatography $A$, 1359, 35-43. http://dx.doi.org/10.1016/j.chroma.2014.07.034

[14] Srebnik, S. (2004) Theoretical Investigation of the Imprinting Efficiency of Molecularly Imprinted Polymers. Chemistry of Materials, 16, 883-888. http://pubs.acs.org/doi/abs/10.1021/cm034705m?journalCode=cmatex

[15] Pavel, D. and Lagowski, J. (2005) Computationally Designed Monomers and Polymers for Molecular Imprinting of Theophylline-Part II. Polymer, 46, 7543-7556. http://dx.doi.org/10.1016/j.polymer.2005.05.146

[16] Golker, K., Karlsson, B.C.G., Wiklander, J.G., Rosengren, A.M. and Nicholls, I.A. (2015) Hydrogen Bond Diversity in the Pre-Polymerization Stage Contributes to Morphology and MIP-Template Recognition-MAA versus MMA. European Polymer Journal, 66, 558-568. http://dx.doi.org/10.1016/j.eurpolymj.2015.03.018

[17] Berglund, J., Nicholls, I.A., Lindbladh, C. and Mosbach, K. (1996) Recognition in Molecularly Imprinted Polymer $\alpha_{2}-$ Adrenoreceptor Mimics. Bioorganic \& Medicinal Chemistry Letters, 6, 2237-2242. http://dx.doi.org/10.1016/0960-894X(96)00406-4

[18] Shimoi, K., Okada, H., Furugori, M., Goda, T., Takase, S., Suzuki, M., Hara, Y., Yamamoto, H. and Kinae, N. (1998) Intestinal Absorption of Luteolin and Luteolin 7-O- $\beta$-Glucoside in Rats and Humans. FEBS Letters, 438, 220-224. http://dx.doi.org/10.1016/S0014-5793(98)01304-0

[19] Lopez-Lazaro, M. (2009) Distribution and Biological Activities of the Flavonoid Luteolin. Mini-Reviews in Medicinal Chemistry, 9, 31-59. http://dx.doi.org/10.2174/138955709787001712

[20] Ulubelen, A., Miski, M., Neuman, P. and Mabry, T.J. (1979) Flavonoids of Salvia tomentosa (Labiatae). Journal of Natural Products, 42, 261-263. http://pubs.acs.org/doi/abs/10.1021/np50003a002

[21] Andersson, H.S. and Nicholls, I.A. (1997) Spectroscopic Evaluation of Molecular Imprinting Polymerization Systems. Bioorganic Chemistry, 25, 203-211. http://dx.doi.org/10.1006/bioo.1997.1067

[22] Pappalardo, A., Amato, M.E., Ballistreri, F.P., Fragola, V.L.P., Tomaselli, G.A., Toscano, R.M. and Sfrazzetto, G.T. (2013) Binding of Reactive Organophosphate by Oximes via Hydrogen Bond. Journal of Chemical Scinces, 125, 869- 
873. http://link.springer.com/article/10.1007/s12039-013-0463-1 http://dx.doi.org/10.1007/s12039-013-0463-1

[23] Wang, X.G., Lin, K.S.K., Chan, J.C.C. and Cheng, S. (2005) Direct Synthesis and Catalytic Applications of Ordered Large Pore Aminopropyl-Functionalized SBA-15 Mesoporous Materials. Journal of Physical Chemistry B, 109, 17631769. http://pubs.acs.org/doi/abs/10.1021/jp045798d http://dx.doi.org/10.1021/jp045798d

[24] Urraca, J.L., Carbajo, M.C., Torralvo, M.J., González-Vázquez, J., Orellana, G. and Moreno-Bondi, M.C. (2008) Effect of the Template and Functional Monomer on the Textural Properties of Molecularly Imprinted Polymers. Biosensors and Bioelectronics, 24, 155-161. http://dx.doi.org/10.1016/j.bios.2008.04.004

[25] Cleland, D., Olsson, G.D., Karlsson, B.C.G., Nicholls, I.A. and McCluskey, A. (2014) Molecular Dynamic Approaches to the Design and Synthesis of PCB Targeting Molecularly Imprinted Polymers: Interference to Monomer-Template Interactions in Imprinting of 1,2,3-Trichlorobenzene. Organic \& Biomolecular Chemistry, 12, 844-853. http://dx.doi.org/10.1039/C3OB42399A 